\title{
A DIMENSION GAP FOR CONTINUED FRACTIONS WITH INDEPENDENT DIGITS - THE NON STATIONARY CASE
}

\author{
ARIEL RAPAPORT
}

\begin{abstract}
We show there exists a constant $0<c_{0}<1$ such that the dimension of every measure on $[0,1]$, which makes the digits in the continued fraction expansion independent, is at most $1-c_{0}$. This extends a result of Kifer, Peres and Weiss from 2001, which established this under the additional assumption of stationarity. For $k \geq 1$ we prove an analogues statement for measures under which the digits form a $*$-mixing $k$-step Markov chain. This is also generalized to the case of $f$-expansions. In addition, we construct for each $k$ a measure, which makes the continued fraction digits a stationary and *-mixing $k$-step Markov chain, with dimension at least $1-2^{3-k}$.
\end{abstract}

\section{INTRODUCTION}

Let $X$ denote the set of irrational numbers in $(0,1)$. It is well known each $x \in X$ has a unique continued fraction expansion of the form

$$
x=\frac{1}{A_{1}(x)+\frac{1}{A_{2}(x)+\frac{1}{A_{3}(x)+\cdots}},},
$$

where $A_{1}(x), A_{2}(x), \ldots$ are positive integers. Given a probability measure $\nu$ on $X$, each $A_{n}$ defines a random variable on $(X, \nu)$ and the digits $\left\{A_{n}\right\}_{n=1}^{\infty}$ form a discrete time stochastic process.

In 1966, Chatterji [Ch has shown every probability measure $\nu$ on $[0,1]$, which makes the digits in the continued fraction expansion independent variables, is singular with respect to the Lebesgue measure. In 2001, Kifer, Peres and Weiss KPW] have proven that $\operatorname{dim}_{H} \nu \leq 1-c$, if in addition the digits are identically distributed. Here $0<c<1$ is a global constant, independent of $\nu$, and $\operatorname{dim}_{H} \nu$ denotes the Hausdorff dimension of $\nu$, which is defined in Section 2 below. In this paper we show the result from [KPW] remains true, even if the digits are independent but not necessarily identically distributed.

Date: March 9, 2017.

2000 Mathematics Subject Classification. Primary: 11K55, Secondary: 37C45.

Key words and phrases. Continued fractions, Hausdorff dimension.

Supported by ERC grant 306494. 
Assuming $A_{1}, A_{2}, \ldots$ are i.i.d. with $\mathbb{E}\left[\log A_{1}\right]<\infty$ and $H\left(A_{1}\right)<\infty$, where $H\left(A_{1}\right)$ is the entropy of $A_{1}$, Kinney and Pitcher $[\mathrm{KP}]$ have proven that

$$
\operatorname{dim}_{H} \nu=\frac{H\left(A_{1}\right)}{-\int_{0}^{1} \log x^{2} d \nu} .
$$

The Gauss measure

$$
\mu_{G}(E)=\frac{1}{\log 2} \int_{E} \frac{d x}{1+x}
$$

is the unique equilibrium state of the Gauss map $T x=\frac{1}{x}(\bmod 1)$ with respect to the function $x \rightarrow \log x^{2}$. This follows from the thermodynamic formalism approach of Walters Wa1. Hence under the i.i.d. assumption

$$
0=\int_{0}^{1} \log x^{2} d \mu_{G}(x)+h_{\mu_{G}}(T)>\int_{0}^{1} \log x^{2} d \nu(x)+h_{\nu}(T),
$$

where $h_{\eta}(T)$ is the entropy of $T$ with respect to a $T$-invariant measure $\eta$. Since $h_{\nu}(T)=H\left(A_{1}\right)$, we get from (1.1) that $\operatorname{dim}_{H} \nu<1$ in this case. When $A_{1}, A_{2}, \ldots$ are not identically distributed the formula (1.1) is no longer valid, and so it is not even clear that $\operatorname{dim}_{H} \nu$ is strictly less than 1 . As mentioned above, we shall show that there exists a global constant $c_{0}>0$ such that $\operatorname{dim}_{H} \nu \leq 1-c_{0}$, assuming $A_{1}, A_{2}, \ldots$ are independent.

We actually prove more generally that for every integer $k \geq 0$ there exists $0<$ $c_{k}<1$, which depends only on $k$, such that $\operatorname{dim}_{H} \nu \leq 1-c_{k}$ if the digits form a $k$-step Markov chain which is $*$-mixing. This is the main result of this paper. The *-mixing condition was introduced in [BHK], and is a bit less restrictive than the more familiar $\psi$-mixing condition. The definitions are given in Section 2 In the last section we generalize our main result to the case of $f$-expansions.

Given $k \geq 0$ it was shown in $\mathrm{KPW}$ that there exists $0<c_{k}^{\prime}<1$, for which $\operatorname{dim}_{H} \nu \leq 1-c_{k}^{\prime}$ whenever $\nu$ makes the digits a stationary and ergodic $k$-step Markov chain. Our proof is a modification of the argument given there for this result. We shall also construct for each $k$ a measure $\nu_{k}$, under which the digits form a stationary and $\psi$-mixing $k$-step Markov chain, with $\operatorname{dim}_{H} \nu_{k} \geq 1-2^{3-k}$. This of course shows $c_{k}$ and $c_{k}^{\prime}$ are at most $2^{3-k}$.

The rest of the paper is organized as follows. In Section 2 we give some necessary definitions and state our results. In Section 3 we establish a uniform bound on the dimension of subsets of $X$, which are defined via certain digit frequencies. This is the key ingredient in the proof of our main result, which is carried out in Section 4. In Section 5 we construct the measures $\nu_{k}$ mentioned above. In Section 6 we generalize our main result to the setup of $f$-expansions.

Acknowledgement. This paper is a part of the author's PhD thesis conducted at the Hebrew University of Jerusalem. I would like to thank my advisor Professor 
Yuri Kifer, for suggesting to me the problem studied in this paper, and for many helpful discussions.

\section{Preliminaries And Results}

First, we define the mixing conditions mentioned above. Given random variables $\left\{A_{i}\right\}_{i \in I}$, all defined on the same probability space, denote by $\sigma\left\{A_{i}\right\}_{i \in I}$ the smallest $\sigma$-algebra with respect to which each $A_{i}$ is measurable.

Definition 2.1. A sequence of random variables $\left\{A_{n}\right\}_{n=1}^{\infty}$ is called $*$-mixing if there exist an integer $N \geq 1$ and a real valued function $f$, defined on the integers $n \geq N$, such that

- $f$ is non-increasing with $\lim _{n} f(n)=0$, and

- if $n \geq N, m \geq 1, E \in \sigma\left\{A_{1}, \ldots, A_{m}\right\}$ and $F \in \sigma\left\{A_{m+n}, A_{m+n+1}, \ldots\right\}$ then

$$
|\mathbb{P}(E \cap F)-\mathbb{P}(E) \mathbb{P}(F)| \leq f(n) \mathbb{P}(E) \mathbb{P}(F) .
$$

If such an $f$ exists for $N=1$ the sequence is said to be $\psi$-mixing.

Remark 2.2. A sequence of independent random variables is clearly $\psi$-mixing. It is not hard to show that the $\psi$-mixing condition is satisfied for a finite state Markov chain $\left\{A_{n}\right\}_{n=1}^{\infty}$, with state space $S$, for which

$$
\inf \left\{\mathbb{P}\left(A_{n+1}=j \mid A_{n}=i\right): n \geq 1 \text { and } i, j \in S\right\}>0 .
$$

Examples of $*$-mixing countable state Markov chains can be found in Section 3 of $[\mathrm{BHK}$. Another important example of a $\psi$-mixing sequence is obtained by the continued fraction digits with respect to the Gauss measure $\mu_{G}$ (see [Ad] or [He]).

Set $X=(0,1) \backslash \mathbb{Q}$ and for each $x \in X$ and $i \geq 1$ let $\alpha_{i}(x) \in \mathbb{N}:=\{1,2, \ldots\}$ be the $i$ 'th digit in the continued fraction expansion of $x$, i.e.

$$
x=\frac{1}{\alpha_{1}(x)+\frac{1}{\alpha_{2}(x)+\frac{1}{\alpha_{3}(x)+\cdots}}} .
$$

Given $a_{1}, a_{2}, \ldots \in \mathbb{N}$ denote by $\left[a_{1}, a_{2}, \ldots\right]$ the unique $x \in X$ with $\alpha_{i}(x)=a_{i}$ for $i \geq 1$. For $E \subset X$ write $\operatorname{dim}_{H}(E)$ for the Hausdorff dimension of $E$. Given a Borel probability measure $\nu$ on $X$ its Hausdorff dimension is defined by

$$
\operatorname{dim}_{H}(\nu)=\inf \left\{\operatorname{dim}_{H}(E): E \subset X \text { is a Borel set with } \nu(E)=1\right\} .
$$

The following theorem is our main result.

Theorem 2.3. Let $\left\{A_{n}\right\}_{n=1}^{\infty}$ be $\mathbb{N}$-valued random variables and let $k \geq 0$. Assume $\left\{A_{n}\right\}_{n=1}^{\infty}$ is a k-step Markov chain (when $k=0$ this means $A_{1}, A_{2}, \ldots$ are independent) which is $*$-mixing. Let $\nu$ be the distribution of the random variable $\left[A_{1}, A_{2}, \ldots\right]$. Then $\operatorname{dim}_{H}(\nu) \leq 1-c_{k}$, where $0<c_{k}<1$ is a constant depending only on $k$. 
Remark 2.4. As mentioned in the introduction, it was shown in [KPW] that there exists $0<c_{k}^{\prime}<1$, for which $\operatorname{dim}_{H} \nu \leq 1-c_{k}^{\prime}$ whenever $\nu$ makes the continued fraction digits a stationary and ergodic $k$-step Markov chain.

It might be desirable to estimate $c_{k}$ and $c_{k}^{\prime}$. The next claim shows these constants are at most $2^{3-k}$.

Claim 2.5. For each $k \geq 3$ there exits an $\mathbb{N}$-valued $k$-step stationary and $\psi$-mixing Markov chain $\left\{A_{n}\right\}_{n=1}^{\infty}$ with $\operatorname{dim}_{H}(\nu) \geq 1-2^{3-k}$, where $\nu$ is the distribution of $\left[A_{1}, A_{2}, \ldots\right]$.

The main ingredient in the proof of Theorem 2.3 is Theorem 2.6 stated below, for which we need some more notations. Let $T: X \rightarrow X$ be the Gauss map, which is defined by

$$
T x=\frac{1}{x}(\bmod 1) \text { for } x \in X .
$$

Denote by $\mu_{G}$ the Gauss measure, which satisfies

$$
\mu_{G}(E)=\frac{1}{\log 2} \int_{E} \frac{d x}{1+x} \text { for every Borel set } E \subset X .
$$

It is well known that $\mu_{G}$ is invariant and ergodic with respect to $T$. For $\left(a_{1}, \ldots, a_{k}\right)=$ $\mathbf{a} \in \mathbb{N}^{k}$ set

$$
I_{\mathbf{a}}=\left\{x \in X: \alpha_{i}(x)=a_{i} \text { for each } 1 \leq i \leq k\right\},
$$

and define $\mathbb{I}_{\mathbf{a}}: X \rightarrow\{0,1\}$ by

$$
\mathbb{I}_{\mathbf{a}}(x)=\left\{\begin{array}{ll}
1 & , \text { if } x \in I_{\mathbf{a}} \\
0 & , \text { if } x \notin I_{\mathbf{a}}
\end{array} \text { for } x \in X .\right.
$$

Given $L>1$ denote by $\mathcal{Q}_{L}$ the set of maps $q: \mathbb{N} \rightarrow \mathbb{N}$ with

$$
q(n+1)>q(n) \text { for each } n \in \mathbb{N}
$$

and

$$
\liminf _{n \rightarrow \infty} \frac{q(n)}{n}<L .
$$

For $q \in \mathcal{Q}_{L}, \mathbf{a} \in \cup_{k=1}^{\infty} \mathbb{N}^{k}$ and $\delta>0$ define

$$
\Gamma_{q, \mathbf{a}}^{\delta}=\left\{x \in X: \liminf _{n \rightarrow \infty}\left|\frac{1}{n} \sum_{i=1}^{n} \mathbb{I}_{\mathbf{a}}\left(T^{q(i)} x\right)-\mu_{G}\left(I_{\mathbf{a}}\right)\right|>\delta\right\} .
$$

Theorem 2.6. For every $L>1$ and $\delta>0$ there exists $0<c_{L, \delta}<1$ with

$$
\sup \left\{\operatorname{dim}_{H}\left(\Gamma_{q, \mathbf{a}}^{\delta}\right): q \in \mathcal{Q}_{L}, \mathbf{a} \in \cup_{k=1}^{\infty} \mathbb{N}^{k}\right\} \leq 1-c_{L, \delta} .
$$

Remark 2.7. The proof of theorem 2.6 resembles the proof of the main result (Theorem 2.1) of [KPW]. There an upper bound, which depends only on $\delta$, is obtained 
for the dimension of sets of the form

$$
\left.\left\{x \in X: \limsup _{n \rightarrow \infty}\left|\frac{1}{n} \sum_{i=1}^{n} \mathbb{I}_{\mathbf{a}}\left(T^{i} x\right)-\mu_{G}\left(I_{\mathbf{a}}\right)\right|>\delta\right\}\right\} .
$$

Here we need to consider the families $\mathcal{Q}_{L}$, and the more general averages

$$
\frac{1}{n} \sum_{i=1}^{n} \mathbb{I}_{\mathbf{a}}\left(T^{q(i)} x\right)
$$

due to the lack of stationarity. As a result we must define $\Gamma_{q, \mathbf{a}}^{\delta}$ with liminf, as opposed to the sets (2.1) which are defined with limsup.

\section{Proof of Theorem 2.6}

The following large deviations estimate will be needed. Its proof is almost identical to the proof of Lemma 3.1 from [KPW, but we include it here for completeness.

Lemma 3.1. Suppose $\mathbf{S}=\left\{\eta_{n}\right\}_{n=1}^{\infty}$ is a stationary and $*$-mixing sequence of random variables. Let $k \geq 1$ and $F: \mathbb{R}^{k} \rightarrow\{0,1\}$, set

$$
p=\mathbb{P}\left\{F\left(\eta_{1}, \ldots, \eta_{k}\right)=1\right\},
$$

and let $q: \mathbb{N} \rightarrow \mathbb{N}$ be strictly increasing. Then for every $\delta>0$ there exists a constant $M=M(\mathbf{S}, \delta, k)>1$, independent of $q$ and $F$, such that for every $n \geq 1$,

$$
\mathbb{P}\left\{\left|\frac{1}{n} \sum_{i=1}^{n} F\left(\eta_{q(i)}, \ldots, \eta_{q(i)+k-1}\right)-p\right|>\delta\right\} \leq M \cdot e^{-n / M} .
$$

Proof. Fix $\delta>0$, then since $\mathbf{S}$ is $*$-mixing there exists $M \in \mathbb{N}$ with

$$
\left|\mathbb{P}\left(E_{1} \cap E_{2}\right)-\mathbb{P}\left(E_{1}\right) \mathbb{P}\left(E_{2}\right)\right| \leq \frac{\delta^{2}}{2} \mathbb{P}\left(E_{1}\right) \mathbb{P}\left(E_{2}\right)
$$

for each $m \geq 1, E_{1} \in \sigma\left\{\eta_{1}, \ldots, \eta_{m+k-1}\right\}$ and $E_{2} \in \sigma\left\{\eta_{m+M}, \eta_{m+M+1}, \ldots\right\}$. For $i \geq 1$ set $\xi_{i}=F\left(\eta_{i}, \ldots, \eta_{i+k-1}\right)$, fix $n \geq M$, and write

$$
A_{n}=\left\{\left|\frac{1}{n} \sum_{i=1}^{n} \xi_{q(i)}-p\right|>\delta\right\} .
$$

Let $N$ be the integral part of $n / M$, and for $1 \leq j \leq M$ set

$$
B_{n, j}=\left\{\left|\frac{1}{N} \sum_{i=0}^{N-1} \xi_{q(j+i M)}-p\right|>\delta-\frac{1}{N}\right\} .
$$

Clearly $A_{n} \subset \cup_{j=1}^{M} B_{n, j}$, hence

$$
\mathbb{P}\left(A_{n}\right) \leq \sum_{j=1}^{M} \mathbb{P}\left(B_{n, j}\right) .
$$


Fix $1 \leq j \leq M$, and for $\epsilon_{0}, \ldots, \epsilon_{N-1} \in\{0,1\}$ write

$$
\mathcal{C}_{\epsilon_{0}, \ldots, \epsilon_{N-1}}=\left\{\xi_{q(j+i M)}=\epsilon_{i} \text { for each } 0 \leq i<N\right\} .
$$

Let $\zeta_{0}, \zeta_{1}, \ldots$ be independent $\{0,1\}$-valued random variables with mean $p$. Since $q$ is strictly increasing it follows easily from (3.1) that,

$$
\begin{aligned}
\mathbb{P}\left(\mathcal{C}_{\epsilon_{0}, \ldots, \epsilon_{N-1}}\right) & \leq\left(1+\frac{\delta^{2}}{2}\right)^{N} \cdot \prod_{i=0}^{N-1} \mathbb{P}\left\{\xi_{q(j+i M)}=\epsilon_{i}\right\} \\
& \leq e^{\delta^{2} N / 2} \cdot \mathbb{P}\left\{\zeta_{i}=\epsilon_{i} \text { for each } 0 \leq i<N\right\} .
\end{aligned}
$$

Set $Z=\sum_{i=0}^{N-1} \zeta_{i}$, then $Z$ is a binomial random variable with parameters $N$ and $p$, and

$$
\begin{aligned}
\mathbb{P}\left(B_{n, j}\right) & =\sum_{\left|\sum_{i=0}^{N-1} \epsilon_{i}-N p\right|>N \delta-1} \mathbb{P}\left(\mathcal{C}_{\epsilon_{0}, \ldots, \epsilon_{N-1}}\right) \\
& \leq e^{\delta^{2} N / 2} \cdot \mathbb{P}\{|Z-N p|>N \delta-1\} .
\end{aligned}
$$

By the exponential estimate for the binomial distribution (see e.g. Cor. A.1.7 in [AS]) we have for $N \geq 4 / \delta$,

$$
\mathbb{P}\{|Z-N p|>N \delta-1\} \leq 2 e^{-N \delta^{2}} .
$$

This together with (3.3) gives,

$$
\mathbb{P}\left(B_{n, j}\right) \leq 2 e^{-\delta^{2} N / 2} \text { for each } 1 \leq j \leq M .
$$

The lemma now follows from (3.2).

As mentioned in Remark 2.2 the sequence $\left\{\alpha_{i}\right\}_{i=1}^{\infty}$ is $\psi$-mixing with respect to $\mu_{G}$. From this and Lemma 3.1 we get the following corollary.

Corollary 3.2. Given $k \geq 1$ and $\delta>0$ there exists a constant $M=M(\delta, k)>1$, such that for every strictly increasing $q: \mathbb{N} \rightarrow \mathbb{N}, \mathbf{a} \in \mathbb{N}^{k}$ and $n \geq 1$,

$$
\mu_{G}\left\{x \in X:\left|\frac{1}{n} \sum_{i=1}^{n} \mathbb{I}_{\mathbf{a}}\left(T^{q(i)} x\right)-\mu_{G}\left(I_{\mathbf{a}}\right)\right|>\delta\right\} \leq M \cdot e^{-n / M} .
$$

Given $x \in X$ and $n \geq 1$ write $J_{n}(x)=I_{\left(\alpha_{1}(x), \ldots, \alpha_{n}(x)\right)}$. Let $\mathcal{L}$ be the Lebesgue measure, and write $|I|=\mathcal{L}(I)$ for $I \subset X$. For $s \geq 0$ let $\mathcal{H}^{s}$ be the $s$-dimensional Hausdorff measure on $X$. For $\eta>0$ and $E \subset X$ write

$$
\mathcal{H}_{\eta}^{s}(E)=\inf \left\{\sum_{i=1}^{\infty}\left|I_{i}\right|^{s}: E \subset \cup_{i=1}^{\infty} I_{i} \text { and }\left|I_{i}\right| \leq \eta\right\},
$$

then

$$
\lim _{\eta \downarrow 0} \mathcal{H}_{\eta}^{s}(E)=\mathcal{H}^{s}(E)
$$


Given $n \geq 1$ write

$$
\beta_{n}=\sup \left\{\left|I_{\mathbf{a}}\right|: \mathbf{a} \in \mathbb{N}^{n}\right\},
$$

then $\beta_{n} \stackrel{n}{\rightarrow} 0$.

Proof of Theorem [2.6. Let $\delta>0, L>1, q \in \mathcal{Q}_{L}, k \geq 1$ and $\mathbf{a} \in \mathbb{N}^{k}$. Given $\lambda>0$ set,

$$
\mathcal{E}_{\lambda}:=\cap_{j=1}^{\infty} \cup_{n=j}^{\infty}\left\{x \in X:\left|J_{n}(x)\right| \leq e^{-\lambda n}\right\} .
$$

By Theorem 4.1 in [KPW] there exists $\lambda>0$ with $\operatorname{dim}_{H} \mathcal{E}_{\lambda}<1$. For $N \geq 1$ set

$$
\Gamma_{q, \mathbf{a}}^{\delta, N}:=\left\{x \in X: \begin{array}{c}
\left|\frac{1}{n} \sum_{i=1}^{n} \mathbb{I}_{\mathbf{a}}\left(T^{q(i)} x\right)-\mu_{G}\left(I_{\mathbf{a}}\right)\right|>\delta, \\
\left|J_{q(n)+k}(x)\right| \geq e^{-\lambda(q(n)+k)}
\end{array} \quad \text { for all } n \geq N\right\},
$$

then

$$
\Gamma_{q, \mathbf{a}}^{\delta} \backslash \mathcal{E}_{\lambda} \subset \cup_{N=1}^{\infty} \Gamma_{q, \mathbf{a}}^{\delta, N} .
$$

Fix $N \geq 1$ and for $n \geq 1$ set

$$
\Upsilon_{q, \mathbf{a}}^{\delta, n}:=\left\{x \in X: \begin{array}{c}
\left|\frac{1}{n} \sum_{i=1}^{n} \mathbb{I}_{\mathbf{a}}\left(T^{q(i)} x\right)-\mu_{G}\left(I_{\mathbf{a}}\right)\right|>\delta \\
\left|J_{q(n)+k}(x)\right| \geq e^{-\lambda(q(n)+k)}
\end{array}\right\},
$$

then $\Gamma_{q, \mathbf{a}}^{\delta, N} \subset \Upsilon_{q, \mathbf{a}}^{\delta, n}$ for all $n \geq N$.

Let $M=M(\delta, k)>1$ be as in Corollary 3.2, set $s=1-\frac{1}{\lambda L M}$ and let $\eta>0$. From $q \in \mathcal{Q}_{L}$ we get $\liminf _{n \rightarrow \infty} \frac{q(n)}{n}<L$. From this and $\beta_{n} \stackrel{n}{\rightarrow} 0$ it follows that there exists $n \geq N$ such that $\beta_{n}<\eta$ and $q(n)<n L$. By the definition of $\Upsilon_{q, \mathbf{a}}^{\delta, n}$ there exists $B_{n} \subset \mathbb{N}^{q(n)+k}$ with $\Upsilon_{q, \mathbf{a}}^{\delta, n}=\cup_{\mathbf{b} \in B_{n}} I_{\mathbf{b}}$. From Corollary 3.2 we get

$$
\mu_{G}\left(\Upsilon_{q, \mathbf{a}}^{\delta, n}\right) \leq M \cdot e^{-n / M} .
$$

Since

$$
r:=\min _{x \in[0,1]} \frac{d \mu_{G}}{d \mathcal{L}}(x)>0,
$$

it follows

$$
\sum_{\mathbf{b} \in B_{n}}\left|I_{\mathbf{b}}\right|=\mathcal{L}\left(\Upsilon_{q, \mathbf{a}}^{\delta, n}\right) \leq r^{-1} \cdot \mu_{G}\left(\Upsilon_{q, \mathbf{a}}^{\delta, n}\right) \leq r^{-1} M \cdot e^{-n / M}
$$

From

$$
\Gamma_{q, \mathbf{a}}^{\delta, N} \subset \Upsilon_{q, \mathbf{a}}^{\delta, n}=\cup_{\mathbf{b} \in B_{n}} I_{\mathbf{b}}
$$

and since $\left|I_{\mathbf{b}}\right| \leq \beta_{n}<\eta$ for every $\mathbf{b} \in B_{n}$,

$$
\mathcal{H}_{\eta}^{s}\left(\Gamma_{q, \mathbf{a}}^{\delta, N}\right) \leq \sum_{\mathbf{b} \in B_{n}}\left|I_{\mathbf{b}}\right|^{s} \leq\left(\inf _{\mathbf{b} \in B_{n}}\left|I_{\mathbf{b}}\right|\right)^{s-1} \cdot \sum_{\mathbf{b} \in B_{n}}\left|I_{\mathbf{b}}\right| .
$$

By the definition of $\Upsilon_{q, \mathbf{a}}^{\delta, n}$,

$$
\left|I_{\mathbf{b}}\right| \geq e^{-\lambda(q(n)+k)} \text { for every } \mathbf{b} \in B_{n} .
$$


Hence from (3.6), (3.5), $q(n)<n L$ and $s=1-\frac{1}{\lambda L M}$,

$$
\begin{aligned}
\mathcal{H}_{\eta}^{s}\left(\Gamma_{q, \mathbf{a}}^{\delta, N}\right) \leq e^{\lambda(q(n)+k)(1-s)} & \cdot r^{-1} M \cdot e^{-n / M} \\
& \leq r^{-1} M e^{\lambda k} \cdot \exp \left(n\left(\lambda L(1-s)-M^{-1}\right)\right)=r^{-1} M e^{\lambda k} .
\end{aligned}
$$

As this holds for every $\eta>0$

$$
\mathcal{H}^{s}\left(\Gamma_{q, \mathbf{a}}^{\delta, N}\right)=\lim _{\eta \downarrow 0} \mathcal{H}_{\eta}^{s}\left(\Gamma_{q, \mathbf{a}}^{\delta, N}\right) \leq r^{-1} M e^{\lambda k}<\infty,
$$

and so

$$
\operatorname{dim}_{H}\left(\Gamma_{q, \mathbf{a}}^{\delta, N}\right) \leq s=1-\frac{1}{\lambda L M} .
$$

As this holds for every $N \geq 1$ it follows from (3.4) that,

$$
\operatorname{dim}_{H}\left(\Gamma_{q, \mathbf{a}}^{\delta} \backslash \mathcal{E}_{\lambda}\right) \leq \sup _{N \geq 1} \operatorname{dim}_{H}\left(\Gamma_{q, \mathbf{a}}^{\delta, N}\right) \leq 1-\frac{1}{\lambda L \cdot M(\delta, k)} .
$$

We shall now complete the proof of the theorem. We continue to fix $\delta>0$ and $L>1$. Let

$$
k_{\delta}=\inf \left\{k \geq 1: \sup _{\mathbf{a} \in \mathbb{N}^{k}} \mu_{G}\left(I_{\mathbf{a}}\right)<\frac{\delta}{2}\right\},
$$

then clearly $k_{\delta}<\infty$. For $q \in \mathcal{Q}_{L}, k \geq k_{\delta}$ and $\left(a_{1}, \ldots, a_{k}\right)=\mathbf{a} \in \mathbb{N}^{k}$,

$$
\Gamma_{q, \mathbf{a}}^{\delta / 2} \supset\left\{x \in X: \liminf _{n \rightarrow \infty} \frac{1}{n} \sum_{i=1}^{n} \mathbb{I}_{\mathbf{a}}\left(T^{q(i)} x\right)>\delta\right\} \supset \Gamma_{q, \mathbf{a}}^{\delta} .
$$

Set $\mathbf{a}_{\delta}=\left(a_{1}, \ldots, a_{k_{\delta}}\right)$, then since $\mathbb{I}_{\mathbf{a}_{\delta}} \geq \mathbb{I}_{\mathbf{a}}$ it follows from (3.8) that $\Gamma_{q, \mathbf{a}_{\delta}}^{\delta / 2} \supset \Gamma_{q, \mathbf{a}}^{\delta}$, and so

$$
\operatorname{dim}_{H}\left(\Gamma_{q, \mathbf{a}_{\delta}}^{\delta / 2}\right) \geq \operatorname{dim}_{H}\left(\Gamma_{q, \mathbf{a}}^{\delta}\right)
$$

This together with (3.7) gives

$$
\begin{aligned}
\sup \left\{\operatorname{dim}_{H}\left(\Gamma_{q, \mathbf{a}}^{\delta}\right): q \in \mathcal{Q}_{L}, \mathbf{a}\right. & \left.\in \cup_{k=1}^{\infty} \mathbb{N}^{k}\right\} \\
& \leq \max \left\{\operatorname{dim}_{H}\left(\mathcal{E}_{\lambda}\right), \max _{1 \leq k \leq k_{\delta}}\left(1-\frac{1}{\lambda L \cdot M(\delta / 2, k)}\right)\right\}<1,
\end{aligned}
$$

which completes the proof of the theorem.

\section{Proof of the main Result}

Proof of Theorem 2.3. Fix $k \geq 0$, let $\left\{A_{n}\right\}_{n=1}^{\infty}$ an $\mathbb{N}$-valued $k$-step Markov chain which is $*$-mixing, and let $\nu$ be the distribution of $\left[A_{1}, A_{2}, \ldots\right]$. Given words $\mathbf{a} \in \mathbb{N}^{m}$ and $\mathbf{b} \in \mathbb{N}^{l}$ we denote by $\mathbf{a b} \in \mathbb{N}^{m+l}$ their concatenation. As noted in observation 2.2 in [KPW], the continued fraction digits under $\mu_{G}$ do not form a $k$-step Markov chain. It follows that there exist $m \in \mathbb{N}, \mathbf{a} \in \mathbb{N}^{k}, \mathbf{b} \in \mathbb{N}^{m}$ and $c \in \mathbb{N}$ with

$$
\frac{\mu_{G}\left(I_{\mathbf{b a c}}\right)}{\mu_{G}\left(I_{\mathbf{b a}}\right)} \neq \frac{\mu_{G}\left(I_{\mathbf{a c}}\right)}{\mu_{G}\left(I_{\mathbf{a}}\right)},
$$


and so

$$
\delta:=\left|\mu_{G}\left(I_{\mathbf{b a c}}\right)-\frac{\mu_{G}\left(I_{\mathbf{a c}}\right) \cdot \mu_{G}\left(I_{\mathbf{b a}}\right)}{\mu_{G}\left(I_{\mathbf{a}}\right)}\right|>0 .
$$

If $k=0$, i.e. when $A_{1}, A_{2}, \ldots$ are independent, $\mathbf{a}$ is the empty word and $I_{\mathbf{a}}=X$. Let $\mu_{G}\left(I_{\mathbf{a}}\right)>\epsilon>0$ be such that if $p_{1}, p_{2}, p_{3} \in[0,1]$ satisfy

$$
\left|p_{1}-\mu_{G}\left(I_{\mathbf{a c}}\right)\right|,\left|p_{2}-\mu_{G}\left(I_{\mathbf{b a}}\right)\right|,\left|p_{3}-\mu_{G}\left(I_{\mathbf{a}}\right)\right| \leq \epsilon,
$$

then

$$
\left|\frac{p_{1} \cdot p_{2}}{p_{3}}-\frac{\mu_{G}\left(I_{\mathbf{a c}}\right) \cdot \mu_{G}\left(I_{\mathbf{b a}}\right)}{\mu_{G}\left(I_{\mathbf{a}}\right)}\right|<\frac{\delta}{2} .
$$

For each $i \geq 1$ and $\mathbf{d} \in \cup_{k=1}^{\infty} \mathbb{N}^{k}$ denote by $E_{\mathbf{d}, i}$ the event

$$
\left\{A_{i} \ldots A_{i+|\mathbf{d}|-1}=\mathbf{d}\right\} \text {, }
$$

where $|\mathbf{d}|$ stands for the length of $\mathbf{d}$, and set $p_{\mathbf{d}, i}:=\mathbb{P}\left(E_{\mathbf{d}, i}\right)$. Let $\mathbf{d} \in \cup_{k=1}^{\infty} \mathbb{N}^{k}$ and assume

$$
\limsup _{n} \frac{1}{n} \#\left\{1 \leq i \leq n: p_{\mathbf{d}, i}<\mu_{G}\left(I_{\mathbf{d}}\right)-\epsilon\right\}>\frac{1}{10},
$$

then there exists $q \in \mathcal{Q}_{10}$ with

$$
p_{\mathbf{d}, q(i)}<\mu_{G}\left(I_{\mathbf{d}}\right)-\epsilon \text { for all } i \geq 1 .
$$

Since $\left\{A_{n}\right\}_{n=1}^{\infty}$ is $*$-mixing it is evident from the definition that $\left\{1_{E_{\mathbf{d}, q(i)}}\right\}_{i=1}^{\infty}$ is also $*$-mixing, where $1_{E}$ denotes the indicator of the event $E$. By the law of large numbers for sums of $*$-mixing bounded random variables (see Theorem 2 in $[\overline{B H K}]$ ),

$$
\lim _{n} \frac{1}{n} \sum_{i=1}^{n}\left(1_{E_{\mathbf{d}, q(i)}}-p_{\mathbf{d}, q(i)}\right)=0 \text { almost surely . }
$$

Hence for $\nu$-a.e. $x \in X$,

$$
\lim _{n}\left|\frac{1}{n} \sum_{i=1}^{n} \mathbb{I}_{\mathbf{d}}\left(T^{q(i)} x\right)-\frac{1}{n} \sum_{i=1}^{n} p_{\mathbf{d}, q(i)}\right|=0 .
$$

From this and (4.3) we get that for $\nu$-a.e. $x \in X$,

$$
\liminf _{n \rightarrow \infty}\left|\frac{1}{n} \sum_{i=1}^{n} \mathbb{I}_{\mathbf{d}}\left(T^{q(i)} x\right)-\mu_{G}\left(I_{\mathbf{d}}\right)\right|=\liminf _{n \rightarrow \infty}\left|\frac{1}{n} \sum_{i=1}^{n} p_{\mathbf{d}, q(i)}-\mu_{G}\left(I_{\mathbf{d}}\right)\right| \geq \epsilon,
$$

which implies $\nu\left(\Gamma_{q, \mathbf{d}}^{\epsilon / 2}\right)=1$. Now by Theorem 2.6

$$
\operatorname{dim}_{H}(\nu) \leq \operatorname{dim}_{H}\left(\Gamma_{q, \mathbf{d}}^{\epsilon / 2}\right) \leq 1-c_{10, \epsilon / 2} .
$$

In a similar manner it can be shown that $\operatorname{dim}_{H}(\nu) \leq 1-c_{10, \epsilon / 2}$ if

$$
\limsup _{n} \frac{1}{n} \#\left\{1 \leq i \leq n: p_{\mathbf{d}, i}>\mu\left(I_{\mathbf{d}}\right)+\epsilon\right\}>\frac{1}{10} .
$$


It follows that we can assume

$$
\liminf _{n} \frac{1}{n} \#\left\{\begin{array}{c}
\left|p_{\mathbf{b a}, i}-\mu\left(I_{\mathbf{b a}}\right)\right| \leq \epsilon, \\
1 \leq i \leq n: \quad\left|p_{\mathbf{a} c, i+m}-\mu\left(I_{\mathbf{a} c}\right)\right| \leq \epsilon, \\
\left|p_{\mathbf{a}, i+m}-\mu\left(I_{\mathbf{a}}\right)\right| \leq \epsilon
\end{array}\right\}>\frac{1}{10},
$$

and so there exists $q \in \mathcal{Q}_{10}$ with

$$
\left|p_{\mathbf{b a}, q(i)}-\mu_{G}\left(I_{\mathbf{b a}}\right)\right|,\left|p_{\mathbf{a} c, q(i)+m}-\mu_{G}\left(I_{\mathbf{a} c}\right)\right|,\left|p_{\mathbf{a}, q(i)+m}-\mu_{G}\left(I_{\mathbf{a}}\right)\right| \leq \epsilon
$$

for all $i \geq 1$. Since $\left\{A_{n}\right\}_{n=1}^{\infty}$ is a Markov chain of order $k$

$$
p_{\mathbf{b a c}, q(i)}=\frac{p_{\mathbf{b a}, q(i)} \cdot p_{\mathbf{a} c, q(i)+m}}{p_{\mathbf{a}, q(i)+m}} \text { for } i \geq 1,
$$

where $p_{\mathbf{a}, q(i)+m}>0$ by (4.4) and $\mu_{G}\left(I_{\mathbf{a}}\right)>\epsilon$. The sequence $\left\{1_{E_{\mathbf{b a}, q(i)}}\right\}_{i=1}^{\infty}$ is $*$-mixing, so by the law of large numbers for sums of $*$-mixing random variables,

$$
\lim _{n} \frac{1}{n} \sum_{i=1}^{n}\left(1_{E_{\mathbf{b a}, q(i)}}-p_{\mathbf{b a c}, q(i)}\right)=0 \text { almost surely . }
$$

It follows that for $\nu$-a.e. $x \in X$,

$$
\lim _{n}\left|\frac{1}{n} \sum_{i=1}^{n} \mathbb{I}_{\mathbf{b a c} c}\left(T^{q(i)} x\right)-\frac{1}{n} \sum_{i=1}^{n} p_{\mathbf{b a c}, q(i)}\right|=0 .
$$

From this, (4.5), (4.4), (4.2) and (4.1) we get that for $\nu$-a.e. $x \in X$,

$$
\begin{array}{r}
\liminf _{n}\left|\frac{1}{n} \sum_{i=1}^{n} \mathbb{I}_{\mathbf{b a c}}\left(T^{q(i)} x\right)-\mu_{G}\left(I_{\mathbf{b a c}}\right)\right| \\
=\liminf _{n}\left|\frac{1}{n} \sum_{i=1}^{n} p_{\mathbf{b a c}, q(i)}-\mu_{G}\left(I_{\mathbf{b a c}}\right)\right| \\
\geq\left|\mu_{G}\left(I_{\mathbf{b a c}}\right)-\frac{\mu_{G}\left(I_{\mathbf{a c}}\right) \cdot \mu_{G}\left(I_{\mathbf{b a}}\right)}{\mu_{G}\left(I_{\mathbf{a}}\right)}\right| \\
-\limsup _{n} \frac{1}{n} \sum_{i=1}^{n}\left|\frac{p_{\mathbf{b a}, q(i)} \cdot p_{\mathbf{a c}, q(i)+m}}{p_{\mathbf{a}, q(i)+m}}-\frac{\mu_{G}\left(I_{\mathbf{a c}}\right) \cdot \mu_{G}\left(I_{\mathbf{b a}}\right)}{\mu_{G}\left(I_{\mathbf{a}}\right)}\right| \geq \delta / 2 .
\end{array}
$$

Hence $\nu\left(\Gamma_{q, \mathbf{b a c}}^{\delta / 4}\right)=1$, and so by Theorem 2.6

$$
\operatorname{dim}_{H}(\nu) \leq \operatorname{dim}_{H}\left(\Gamma_{q, \mathbf{b a c}}^{\delta / 4}\right) \leq 1-c_{10, \delta / 4} .
$$

This completes the proof of the theorem.

\section{Construction of the measures $\nu_{K}$}

In the proof below we use the notation for the Kolmogorov-Sinai entropy from Chapter 4 of Wa2. In particular the entropy of a Borel probability measure $\theta$ on $X$, with respect to a countable Borel partition $\xi$ of $X$, is denoted by $H_{\theta}(\xi)$. If $\mathcal{F}$ is a sub- $\sigma$-algebra of the Borel $\sigma$-algebra of $X$, then $H_{\theta}(\xi \mid \mathcal{F})$ is the entropy of $\theta$ with 
respect to $\xi$ conditioned on $\mathcal{F}$. If $\theta$ is $T$-invariant the entropy of $T$ with respect to $\theta$ is denoted by $h_{\theta}$. If $\theta$ is also ergodic we write $\gamma_{\theta}$ for the Lyapunov exponent of the system $(X, T, \theta)$, i.e.

$$
\gamma_{\theta}=\int_{X} \log \left|T^{\prime}(x)\right| d \theta(x)=-2 \int_{X} \log x d \theta(x) .
$$

Given $a_{1}, \ldots, a_{m} \in \mathbb{N}$ we denote by $\left[a_{1}, \ldots, a_{m}\right]$ the finite continued fraction which lies in $(0,1)$ and has coefficients $a_{1}, \ldots, a_{m}$, i.e.

$$
\left[a_{1}, \ldots, a_{m}\right]=\frac{1}{a_{1}+\frac{1}{a_{2}+\cdots \frac{1}{a_{m-1}+\frac{1}{a_{m}}}}} .
$$

In order to establish the $\psi$-mixing property in the proof of Claim 2.5 we shall need the following proposition. It follows directly from Theorem 1 in $[\mathrm{Br}]$.

Proposition 5.1. Let $\left\{A_{n}\right\}_{n=1}^{\infty}$ be a stationary and mixing sequence of random variables. Assume there exists a constant $0<C<\infty$ with

$$
C^{-1} \leq \frac{\mathbb{P}(E \cap F)}{\mathbb{P}(E) \mathbb{P}(F)} \leq C
$$

for all $l \geq 1, E \in \sigma\left\{A_{1}, \ldots, A_{l}\right\}$ and $F \in \sigma\left\{A_{l+1}, A_{l+2}, \ldots\right\}$. Then $\left\{A_{n}\right\}_{n=1}^{\infty}$ is $\psi$-mixing.

Proof of Claim 2.5. Fix $k \geq 3$ and for every $\mathbf{a} \in \mathbb{N}^{k}$ and $c \in \mathbb{N}$ set

$$
p_{\mathbf{a}}=\mu_{G}\left(I_{\mathbf{a}}\right) \text { and } p_{\mathbf{a}, c}=\frac{\mu_{G}\left(I_{\mathbf{a} c}\right)}{\mu_{G}\left(I_{\mathbf{a}}\right)} .
$$

Then $\sum_{c \in \mathbb{N}} p_{\mathbf{a}, c}=1$ for each $\mathbf{a} \in \mathbb{N}^{k}$ and $p=\left\{p_{\mathbf{a}}\right\}_{\mathbf{a} \in \mathbb{N}^{k}}$ is a probability vector. Let $\left\{A_{n}\right\}_{n=1}^{\infty}$ be the $k$-step $\mathbb{N}$-valued Markov chain corresponding to the transition probabilities $\left\{p_{\mathbf{a}, c}\right\}_{(\mathbf{a}, c) \in \mathbb{N}^{k+1}}$ and initial distribution $\left\{p_{\mathbf{a}}\right\}_{\mathbf{a} \in \mathbb{N}^{k}}$. For each $\mathbf{b} \in \mathbb{N}^{k-1}$ and $d \in \mathbb{N}$

$$
\sum_{c \in \mathbb{N}} p_{c \mathbf{b}} \cdot p_{c \mathbf{b}, d}=\sum_{c \in \mathbb{N}} \mu_{G}\left(I_{c \mathbf{b}}\right) \cdot \frac{\mu_{G}\left(I_{c \mathbf{b} d}\right)}{\mu_{G}\left(I_{c \mathbf{b}}\right)}=\mu_{G}\left(T^{-1}\left(I_{\mathbf{b} d}\right)\right)=p_{\mathbf{b} d},
$$

hence $\left\{A_{n}\right\}_{n=1}^{\infty}$ is stationary. Considering $\left\{A_{n}\right\}_{n=1}^{\infty}$ as a 1-step Markov chain on the state space $\mathbb{N}^{k}$, it is easy to see it is irreducible and aperiodic. From this and Theorem 8.6 in $\mathrm{Bi}$ it follows $\left\{A_{n}\right\}_{n=1}^{\infty}$ is mixing.

Let us show $\left\{A_{n}\right\}_{n=1}^{\infty}$ is in fact $\psi$-mixing. From (3.22) in chapter 3 of [EW] it follows there exists a constant $1<C<\infty$ with,

$$
C^{-l} \leq \frac{\mu_{G}\left(I_{\left(a_{1}, \ldots, a_{l}\right)}\right)}{\mu_{G}\left(I_{a_{1}}\right) \cdot \ldots \cdot \mu_{G}\left(I_{a_{l}}\right)} \leq C^{l} \text { for } l \geq 1 \text { and } a_{1}, \ldots, a_{l} \in \mathbb{N} .
$$

For $l, m>k,\left(a_{1}, \ldots, a_{l}\right)=\mathbf{a} \in \mathbb{N}^{l}$ and $\left(b_{1}, \ldots, b_{m}\right)=\mathbf{b} \in \mathbb{N}^{m}$ set

$$
R:=\frac{\mathbb{P}\left\{A_{1} \ldots A_{l+m}=\mathbf{a b}\right\}}{\mathbb{P}\left\{A_{1} \ldots A_{l}=\mathbf{a}\right\} \mathbb{P}\left\{A_{1} \ldots A_{m}=\mathbf{b}\right\}},
$$


then

$$
R=\frac{1}{\mu_{G}\left(I_{\left(b_{1}, \ldots, b_{k}\right)}\right)} \cdot \prod_{j=1}^{k} \frac{\mu_{G}\left(I_{\left(a_{l-k+j}, \ldots, a_{l}, b_{1}, \ldots, b_{j}\right)}\right)}{\mu_{G}\left(I_{\left(a_{l-k+j}, \ldots, a_{l}, b_{1}, \ldots, b_{j-1}\right)}\right)} .
$$

This together with (5.1) gives

$$
C^{-2 k(k+1)} \leq R \leq C^{2 k(k+1)} .
$$

From Proposition 5.1, combined with a monotone class argument, it now follows that $\left\{A_{n}\right\}_{n=1}^{\infty}$ is $\psi$-mixing.

Let $\nu$ be the distribution of $\left[A_{1}, A_{2}, \ldots\right]$, then $\nu$ is $T$-invariant and ergodic. In order to prove the claim it remains to show that $\operatorname{dim}_{H} \nu \geq 1-2^{3-k}$. Set

$$
\xi=\left\{I_{c}: c \in \mathbb{N}\right\}
$$

then it is easy to check that

$$
H_{\nu}(\xi)=H_{\mu_{G}}(\xi)<\infty
$$

and

$$
\sum_{c \in \mathbb{N}} \nu\left(I_{c}\right) \log c=\sum_{c \in \mathbb{N}} \mu_{G}\left(I_{c}\right) \log c<\infty,
$$

which shows $h_{\nu}, \gamma_{\nu}, h_{\mu_{G}}$ and $\gamma_{\mu_{G}}$ are all finite. From this and Section 2 of [BH] it follows that

$$
\operatorname{dim}_{H} \nu=\frac{h_{\nu}}{\gamma_{\nu}} \text { and } 1=\operatorname{dim}_{H} \mu_{G}=\frac{h_{\mu_{G}}}{\gamma_{\mu_{G}}} .
$$

Moreover, it is well known

$$
\gamma_{\mu_{G}}=-\frac{2}{\log 2} \int \frac{\log x}{1+x} d x=\frac{\pi^{2}}{6 \log 2}>2 .
$$

By an argument similar to the one given in Theorem 4.27 in Wa2,

$$
h_{\nu}=-\sum_{\mathbf{a} \in \mathbb{N}^{k}} \sum_{c \in \mathbb{N}} p_{\mathbf{a}} p_{\mathbf{a}, c} \log p_{\mathbf{a}, c} .
$$

From this and the definition of conditional entropy,

$$
h_{\nu}=H_{\mu_{G}}\left(\vee_{j=0}^{k} T^{-j} \xi \mid \vee_{j=0}^{k-1} T^{-j} \sigma(\xi)\right) .
$$

Now from Theorems 4.3 and 4.14 in [Wa2],

$$
\begin{aligned}
h_{\nu}=H_{\mu_{G}}\left(\vee_{j=0}^{k} T^{-j} \xi\right)-H_{\mu_{G}}\left(\vee_{j=1}^{k} T^{-j} \xi\right) & \\
& =H_{\mu_{G}}\left(\xi \mid \vee_{j=1}^{k} T^{-j} \sigma(\xi)\right) \geq H_{\mu_{G}}\left(\xi \mid \vee_{j=1}^{\infty} T^{-j} \sigma(\xi)\right)=h_{\mu_{G}} .
\end{aligned}
$$

Assume $k$ is even for the moment, then

$$
\left[a_{1}, \ldots, a_{k}\right] \leq x \leq\left[a_{1}, \ldots, a_{k}+1\right]
$$


for every $\left(a_{1}, \ldots, a_{k}\right)=\mathbf{a}=\mathbb{N}^{k}$ and $x \in I_{\mathbf{a}}$. It follows that,

$$
\begin{gathered}
\gamma_{\nu}-\gamma_{\mu_{G}}=-2 \int_{X} \log x d \nu(x)+2 \int_{X} \log x d \mu_{G}(x) \\
=2 \sum_{\mathbf{a} \in \mathbb{N}^{k}}\left(\int_{I_{\mathbf{a}}} \log \frac{1}{x} d \nu(x)+\int_{I_{\mathbf{a}}} \log x d \mu_{G}(x)\right) \\
\leq 2 \sum_{a_{1}, \ldots, a_{k} \in \mathbb{N}}\left(\int_{I_{\left(a_{1}, \ldots, a_{k}\right)}} \log \frac{1}{\left[a_{1}, \ldots, a_{k}\right]} d \nu(x)+\int_{I_{\left(a_{1}, \ldots, a_{k}\right)}} \log \left[a_{1}, \ldots, a_{k}+1\right] d \mu_{G}(x)\right) \\
=2 \sum_{a_{1}, \ldots, a_{k} \in \mathbb{N}} \mu_{G}\left(I_{\left(a_{1}, \ldots, a_{k}\right)}\right) \cdot \log \frac{\left[a_{1}, \ldots, a_{k}+1\right]}{\left[a_{1}, \ldots, a_{k}\right]} .
\end{gathered}
$$

Fix $a_{1}, \ldots, a_{k} \in \mathbb{N}$, then

$$
\log \frac{\left[a_{1}, \ldots, a_{k}+1\right]}{\left[a_{1}, \ldots, a_{k}\right]} \leq \frac{\left[a_{1}, \ldots, a_{k}+1\right]-\left[a_{1}, \ldots, a_{k}\right]}{\left[a_{1}, \ldots, a_{k}\right]} .
$$

Let $p, q \in \mathbb{N}$ be with $\operatorname{gcd}(p, g)=1$ and $\frac{p}{q}=\left[a_{1}, \ldots, a_{k}\right]$. From inequalities (3.6), (3.7) and (3.14) in [EW] it follows that $q, p \geq 2^{(k-2) / 2}$ and

$$
\left[a_{1}, \ldots, a_{k}+1\right]-\left[a_{1}, \ldots, a_{k}\right] \leq q^{-2}
$$

Hence

$$
\log \frac{\left[a_{1}, \ldots, a_{k}+1\right]}{\left[a_{1}, \ldots, a_{k}\right]} \leq \frac{1 / q^{2}}{p / q}=\frac{1}{p q} \leq 2^{2-k},
$$

and so $\gamma_{\nu}-\gamma_{\mu_{G}} \leq 2^{3-k}$. By exchanging between $\gamma_{\mu_{G}}$ and $\gamma_{\nu}$ it can be shown that $\gamma_{\mu_{G}}-\gamma_{\nu} \leq 2^{3-k}$. From $k \geq 3$ and (5.3) we get $\gamma_{\nu} \geq 1$, hence

$$
1 \leq \frac{\gamma_{\mu_{G}}}{\gamma_{\nu}}+\frac{2^{3-k}}{\gamma_{\nu}} \leq \frac{\gamma_{\mu_{G}}}{\gamma_{\nu}}+2^{3-k}
$$

A similar argument shows (5.5) holds when $k$ is odd. From (5.2), (5.4) and (5.5) we now get

$$
\operatorname{dim}_{H} \nu=\frac{\gamma_{\mu_{G}}}{\gamma_{\nu}} \cdot \frac{h_{\nu}}{\gamma_{\mu_{G}}} \geq\left(1-2^{3-k}\right) \cdot \frac{h_{\mu_{G}}}{\gamma_{\mu_{G}}}=1-2^{3-k},
$$

which completes the proof of the claim.

\section{EXtension of RESUlts FOR $f$-EXPANSIONS}

With almost no change, Theorems 2.3 and 2.6 extend to the more general setup of $f$-expansions, which we now define. Let $M \in\{2,3, \ldots\} \cup\{\infty\}$. Let $f$ be either a strictly decreasing continuous function defined on $[1, M+1]$ with $f(1)=1$ and $f(M+1)=0$, or a strictly increasing continuous function defined on $[0, M]$ with $f(0)=0$ and $f(M)=1$. For $x \in(0,1)$ set $r_{0}(x)=x$ and $r_{i+1}(x)=\left\{f^{-1}\left(r_{i}(x)\right)\right\}$ for $i \geq 0$, where $\{\cdot\}$ denotes the fractional part of a number. Let $X$ be the set of all 
$x \in(0,1)$ with $r_{i}(x) \neq 0$ for every $i \geq 0$, then $(0,1) \backslash X$ is clearly countable. Write

$$
\mathcal{N}=\left\{[y]: y \in f^{-1}(0,1)\right\},
$$

where [.] is the integer part of a number. For $x \in X$ and $i \geq 1$ set

$$
\alpha_{i}(x)=\left[f^{-1}\left(r_{i-1}(x)\right)\right],
$$

then $\alpha_{i}(x) \in \mathcal{N}$. We shall assume that

$$
x=f\left(\alpha_{1}(x)+f\left(\alpha_{2}(x)+f\left(\alpha_{3}(x)+\ldots\right)\right)\right) \text { for all } x \in X,
$$

and call the expression on the right hand side the $f$-expansion of $x$. Regularity conditions on $f$ were given by Rényi [ $[\mathbf{R}$, which ensure that (6.1) is satisfied. The main example of the decreasing case is $f(x)=1 / x$, which leads to the continued fraction expansion, and of the increasing case is $f(x)=x / M$, which leads to the base- $M$ expansion. For more details on $f$-expansions see $[\mathrm{R}],[\mathrm{KP},[\mathrm{He}]$ and the references therein.

We use the notation $I_{\mathbf{a}}$ and $\mathbb{I}_{\mathbf{a}}$, introduced in Section 2, with $X$ and $\alpha_{i}$ as defined in this section and $\mathbf{a} \in \cup_{k=1}^{\infty} \mathcal{N}^{k}$. For $x \in(0,1)$ set $T x=f^{-1} x-\left[f^{-1} x\right]$, then $\alpha_{i}(T x)=\alpha_{i+1}(x)$ for $x \in X$. We shall assume that

(1) the restriction of $T$ to $f(a, a+1)$ is $C^{2}$ for each $a \in \mathcal{N}$;

(2) there exists $\ell \in \mathbb{N}$ and $\beta>0$ with $\left|\left(T^{\ell}\right)^{\prime}(x)\right| \geq \beta$ for all $x \in X$;

(3) there exists $1 \leq Q<\infty$ with $\left|\frac{T^{\prime \prime}(x)}{T^{\prime}(y) T^{\prime}(z)}\right| \leq Q$ for all $a \in \mathcal{N}$ and $x, y, z \in I_{a}$. Then by Theorem 22 in Wa1, there exists an absolutely continuous $T$-invariant mixing probability measure $\mu_{T}$ on $X$, such that $0<\frac{d \mu_{T}}{d \mathcal{L}} \in C[0,1]$. Here, as above, $\mathcal{L}$ is the Lebesgue measure.

For $q \in \mathcal{Q}_{L}$ with $\mathcal{Q}_{L}$ defined in Section $2, \mathbf{a} \in \cup_{k=1}^{\infty} \mathcal{N}^{k}$ and $\delta>0$ let

$$
\Gamma_{q, \mathbf{a}}^{\delta}=\left\{x \in X: \liminf _{n \rightarrow \infty}\left|\frac{1}{n} \sum_{i=1}^{n} \mathbb{I}_{\mathbf{a}}\left(T^{q(i)} x\right)-\mu_{T}\left(I_{\mathbf{a}}\right)\right|>\delta\right\} .
$$

The following theorem is an analogue of Theorem 2.6 and can be proven in exactly the same manner.

Theorem 6.1. Suppose that $T$ satisfies conditions (1)-(3) and assume, in addition, that for some $t<1$,

$$
\sup _{x \in X} \sum_{y: T y=x}\left|T^{\prime}(y)\right|^{-t}<\infty .
$$

Then for every $L>1$ and $\delta>0$ there exists $0<c_{f, L, \delta}<1$ with

$$
\sup \left\{\operatorname{dim}_{H}\left(\Gamma_{q, \mathbf{a}}^{\delta}\right): q \in \mathcal{Q}_{L}, \mathbf{a} \in \cup_{k=1}^{\infty} \mathcal{N}^{k}\right\} \leq 1-c_{f, L, \delta} .
$$

Remark 6.2. The condition (6.2) is needed in order to apply Theorem 4.1 from KPW], as we did at the beginning of the proof of Theorem 2.6. Since $\left\{\alpha_{i}\right\}_{i=1}^{\infty}$ 
is a $\psi$-mixing sequence with respect to $\mu_{T}$ (see $[\mathrm{Ad}]$ or $[\mathrm{He}]$ ), the large deviations estimate from Corollary 3.2 is valid for $\mu_{T}$. Now the proof of Theorem 6.1 follows almost verbatim the proof of Theorem 2.6.

An important ingredient in the proof of Theorem 2.3 is the fact that, for any $k \geq 0$, the continued fraction digits under $\mu_{G}$ do not form a $k$-step Markov chain. Hence, in order to generalize Theorem 2.3 to the case of $f$-expansions we shall need the following lemma. For $t \in[0,1]$ set $F(t)=\mu_{T}([0, t])$, and let $S=F \circ T \circ F^{-1}$. Since $F^{\prime}=\frac{d \mu_{T}}{d \mathcal{L}} \in C[0,1]$ with $\frac{d \mu_{T}}{d \mathcal{L}}>0, F$ is a diffeomorphism of $[0,1]$ onto itself. Given $a \in \mathcal{N}$ write $\widetilde{I_{a}}:=f(a, a+1)$.

Lemma 6.3. Assume the digits $\left\{\alpha_{i}\right\}_{i=1}^{\infty}$ of the $f$-expansion are not independent under $\mu_{T}$. Then $\left\{\alpha_{i}\right\}_{i=1}^{\infty}$ do not form a k-step Markov chain under $\mu_{T}$ for any $k \geq 1$.

Proof. Note that $F \mu_{T}=\mathcal{L}$ and $S \mathcal{L}=\mathcal{L}$. From the chain rule it follows that for every $a \in \mathcal{N}$ and $x \in F \widetilde{I}_{a}$,

$$
S^{\prime}(x)=F^{\prime}\left(T F^{-1} x\right) T^{\prime}\left(F^{-1} x\right)\left(F^{\prime}\left(F^{-1} x\right)\right)^{-1},
$$

and so $S^{\prime}$ is continuous on $F \widetilde{I_{a}}$. Let $\beta_{1}: F X \rightarrow \mathcal{N}$ be such that $\beta_{1}(x)=a$ for $a \in \mathcal{N}$ and $x \in F I_{a}$. For $i \geq 1$ set $\beta_{i}=\beta_{1} \circ S^{i-1}$, then $\beta_{i}=\alpha_{i} \circ F^{-1}$. Given $\left(a_{1}, \ldots, a_{l}\right)=\mathbf{a} \in \mathcal{N}^{l}$ let

$$
J_{\mathbf{a}}=\left\{x \in F X: \beta_{i}(x)=a_{i} \text { for } 1 \leq i \leq l\right\},
$$

then $J_{\mathbf{a}}=F I_{\mathbf{a}}$. Note that

$$
\mathcal{L}\left(J_{\mathbf{a}}\right)=\mu_{T}\left(I_{\mathbf{a}}\right) \text { for every } l \geq 1 \text { and } \mathbf{a} \in \mathcal{N}^{l} .
$$

Let $k \geq 1$ and assume by contradiction that $\left\{\alpha_{i}\right\}_{i=1}^{\infty}$ forms a $k$-step Markov chain under $\mu_{T}$. From this, since $\left\{\alpha_{i}\right\}_{i=1}^{\infty}$ are not independent, and from (6.3), it follows that under $\mathcal{L}$ the variables $\left\{\beta_{i}\right\}_{i=1}^{\infty}$ form a stationary $k$-step Markov chain but are not independent. Since $\left\{\beta_{i}\right\}_{i=1}^{\infty}$ is a stationary $k$-step Markov chain,

$$
\mathcal{L}\left\{\beta_{1}=c \mid S^{-1}\left(J_{\mathbf{b}}\right)\right\}=\mathcal{L}\left\{\beta_{1}=c \mid S^{-1}\left(J_{\mathbf{b f}}\right)\right\}
$$

for every $c \in \mathcal{N}, \mathbf{b} \in \mathcal{N}^{k}, l \geq 1$ and $\mathbf{f} \in \mathcal{N}^{l}$. It follows there exist $c \in \mathcal{N}$ and $\mathbf{b}, \mathbf{d} \in \mathcal{N}^{k}$ with,

$$
\mathcal{L}\left\{\beta_{1}=c \mid S^{-1}\left(J_{\mathbf{b}}\right)\right\} \neq \mathcal{L}\left\{\beta_{1}=c \mid S^{-1}\left(J_{\mathbf{d}}\right)\right\},
$$

otherwise it would hold that $\left\{\beta_{i}\right\}_{i=1}^{\infty}$ are independent under $\mathcal{L}$.

It is not hard to see that for $\mathcal{L}$-a.e. $x \in J_{c}$,

$$
\mathcal{L}\left\{\beta_{1}=c \mid \sigma\left\{\beta_{2}, \beta_{3}, \ldots\right\}\right\}(x)=\left(S^{\prime}(x)\right)^{-1},
$$


where the left hand side is the conditional $\mathcal{L}$-probability of the event $\left\{\beta_{1}=c\right\}$ with respect to the $\sigma$-algebra $\sigma\left\{\beta_{2}, \beta_{3}, \ldots\right\}$. Let $\mathbf{a} \in \mathcal{N}^{k}$. Then since $\left\{\beta_{i}\right\}_{i=1}^{\infty}$ is a $k$-step Markov chain under $\mathcal{L}$, it follows for $\mathcal{L}$-a.e. $x \in J_{\text {ca }}$ that

$$
\begin{aligned}
\mathcal{L}\left\{\beta_{1}=c \mid \sigma\left\{\beta_{2}, \beta_{3}, \ldots\right\}\right\} & \\
& =\mathcal{L}\left\{\beta_{1}=c \mid \sigma\left\{\beta_{2}, \ldots, \beta_{k+1}\right\}\right\}(x)=\mathcal{L}\left\{\beta_{1}=c \mid S^{-1}\left(J_{\mathbf{a}}\right)\right\} .
\end{aligned}
$$

This together with (6.5) shows that

$$
\left(S^{\prime}(x)\right)^{-1}=\mathcal{L}\left\{\beta_{1}=c \mid S^{-1}\left(J_{\mathbf{a}}\right)\right\} \text { for } \mathcal{L} \text {-a.e. } x \in J_{c \mathbf{a}} .
$$

Since $S^{\prime}$ is continuous on $F \widetilde{I}_{c}$ and

$$
F\left(\widetilde{I}_{c} \cap X\right)=\cup_{\mathbf{a} \in \mathcal{N}^{k}} J_{c \mathbf{a}},
$$

it follows easily from (6.6) that $S^{\prime}$ must be constant on $F \widetilde{I}_{c}$. On the other hand, by (6.4) and (6.6) this is not possible. We have thus reached a contradiction, which shows that $\left\{\alpha_{i}\right\}_{i=1}^{\infty}$ does not form a $k$-step Markov chain under $\mu_{T}$.

Remark 6.4. In Proposition 7.1 from [KPW] it is shown that $\left\{\alpha_{i}\right\}_{i=1}^{\infty}$ are independent under $\mu_{T}$ if and only if $S$ is linear on $F \widetilde{I}_{a}$ for each $a \in \mathcal{N}$. From this and Lemma 6.3 it follows that if $S$ is not linear on $F \widetilde{I}_{a}$ for some $a \in \mathcal{N}$, then $\left\{\alpha_{i}\right\}_{i=1}^{\infty}$ do not form a $k$-step Markov chain under $\mu_{T}$ for any $k \geq 0$.

The following theorem is an analogue, for the case of $f$-expansions, of Theorem 2.3 above and Corollary 2.3 from [KPW]. It can be derived from Theorem 6.1. Theorem 2.1 in [KPW], and Lemma 6.3. by an argument similar to the one given in the proof of Theorem 2.3. Given $a_{1}, a_{2}, \ldots \in \mathcal{N}$ denote by $\left[a_{1}, a_{2}, \ldots\right]$ the unique $x \in X$ with $\alpha_{i}(x)=a_{i}$ for $i \geq 1$.

Theorem 6.5. Suppose that $T$ satisfies the conditions (1)-(3) and, in addition, that (6.2) holds for some $t<1$. Assume the digits $\left\{\alpha_{i}\right\}_{i=1}^{\infty}$ of the $f$-expansion are not independent under $\mu_{T}$. Let $k \geq 0$ and let $\left\{A_{n}\right\}_{n=1}^{\infty}$ be an $\mathcal{N}$-valued $k$-step Markov chain (when $k=0$ this means $A_{1}, A_{2}, \ldots$ are independent). Assume $\left\{A_{n}\right\}_{n=1}^{\infty}$ is *mixing or that it is stationary and ergodic. Let $\nu$ be the distribution of the random variable $\left[A_{1}, A_{2}, \ldots\right]$. Then $\operatorname{dim}_{H}(\nu) \leq 1-c_{f, k}$, where $0<c_{f, k}<1$ is a constant depending only on $f$ and $k$.

\section{REFERENCES}

[Ad] R. Adler, Continued fractions and Bernoulli trials, in: Ergodic Theory (A Seminar), J. Moser, E. Phillips and S. Varadhan, eds., Courant Inst. of Math. Sci. (Lect. Notes 110), 1975, New York.

[AS] N. Alon, J. Spencer, The Probabilistic Method, Wiley, New York, 1992.

[Bi] P.Billingsley, Probability and Measure, 3rd edn., Wiley, New York 1995. 
[BH] P.Billingsley and I.Henningsen, Hausdorff dimension of some continued-fraction sets. Z. Wahrsch. verw. Geb. 31 (1975), 163-173.

[BHK] J.R.Blum, D.L.Hanson and L.H.Koopmans, On the Strong Law of Large Numbers for a Class of Stochastic Processes. Z. Wahrsch. verw. Geb. 2 (1963), 1-11.

[Br] R.C.Bradley, On the $\psi$-mixing condition for stationary random sequences. Trans. Amer. Math. Soc. 276 (1983) 55-66. MR684493.

[Ch] S.D.Chatterji, Masse, die von regelmässigen Kettenbruchen induziert sind, Math. Ann., vol. 164 (1966), pp. 113-117. MR0193079 (33:1300).

[EW] M.Einsiedler and T.Ward, Ergodic Theory with a View Towards Number Theory (Graduate Texts in Mathematics, 259). Springer, London, 2011.

[He] L.Heinrich, Mixing properties and central limit theorem for a class of non-identical piecewise monotonic $C^{2}$-transformations. Mathematische Nachricht. 181, 185-214 (1996).

[KPW] Y.Kifer, Y.Peres and B.Weiss, A dimension gap for continued fractions with independent digits. Israel J. Math. 124(1), (2001), 61-76.

[KP] J.R.Kinney and T.S.Pitcher, The Dimension of Some Sets Defined in Terms of $f$ expansions. Z. Wahrsch. verw. Geb. 4 (1966), 293-315

[R] A.Rényi, Representations for real numbers and their ergodic properties, Acta Math. Acad. Sci. Hung., 8 (1957), pp. 477-493.

[Wa1] P.Walters, Invariant measures and equilibrium states for some mappings which expand distances. Trans. Amer. Math. Soc. 236 (1975), 121-153.

[Wa2] P.Walters, An introduction to ergodic theory. Springer, Verlag, 2001. 